Review Paper http://ajol.info/index.php/ijbcs http://indexmedicus.afro.who.int

\title{
Etapes et méthodes de formulation d'aliment de volaille : Une synthèse bibliographique
}

\author{
N. BRAH ${ }^{1,2}$, M. F. HOUNDONOUGBO ${ }^{1 *}$ et S. ISSA ${ }^{2}$ \\ ${ }^{1}$ Ecole des Sciences et Techniques de Production Animale, Faculté des Sciences Agronomiques, \\ Université Abomey Calavi, Bénin. \\ ${ }^{2}$ Département Production Animale, Institut National de la Recherche Agronomique du Niger, Niger. \\ *Auteur correspondant ; E-mail : fredericmh@gmail.com
}

\section{RESUME}

L'alimentation joue un rôle déterminant dans la réussite et la rentabilité économique des productions avicoles. Lors de la formulation d'un aliment efficient, son coût et sa qualité nutritionnelle qui permet de couvrir tous les besoins nutritionnels essentiels des volailles doivent être considérés. Ce travail basé sur la collecte des articles, fait la revue des étapes à suivre pour la formulation d'aliment de volaille et décrit les méthodes qui ont été utilisées dans la formulation d'aliment de volaille. Le carré de Pearson, l'équation algébrique simultanée, la méthode matérielle et la méthode essai et erreur sont des méthodes manuelles utilisées pour la formulation d'aliment de volaille. Les programmations linéaire, non linéaire, multi objective et quadratique sont quant à elles les méthodes de programmation mathématique de formulation d'aliment de volaille. Les méthodes manuelles permettent de faire une formulation avec peu d'ingrédient et les méthodes mathématiques permettent de faire une formulation à moindre coût.

(C) 2015 International Formulae Group. All rights reserved.

Mots clés : Aliment de volaille, formulation, étapes, méthodes.

\section{Steps and methods of poultry feed formulation: A review}

\begin{abstract}
Diet plays a key role in the success and the profitability of poultry production. When formulating an efficient food, costs and nutritional quality that can cover all the nutritional requirements of poultry must be considered. This work, based on the collection of articles, is reviewing the steps for poultry feed formulation and the methods that were used in the formulation of poultry feed. Pearson's square, simultaneous algebraic equation, the matrix method and the trial and error method are manual methods used for the formulation of poultry feed. The linear programming, nonlinear, multi objective and quadratic are mathematical programming methods of poultry feed formulation. Manual methods allow a formulation with little ingredient and mathematical methods allow for a lower cost formulation.
\end{abstract}

(0) 2015 International Formulae Group. All rights reserved.

Keywords: Poultry feed, formulation, stages, methods.

\section{INTRODUCTION}

En aviculture, l'alimentation affecte plus la rentabilité économique de l'exploitation que n'importe qu'elle facteur pris individuellement. L'aliment peut représenter $70 \%$ des charges variables de 
production d'œufs ou de chair (Oladokun et Johnson, 2012), d'où l'attention accordée à ce facteur par les acteurs de la chaîne.

Pour maintenir un niveau de profit satisfaisant, la maîtrise des matières premières et des méthodes de formulation sont nécessaires (Afolayan et Afolayan, 2008).

La formulation d'aliment consiste à rassembler plusieurs matières premières disponibles, quantifier leur taux d'incorporation et les mettre ensemble pour former un mélange uniforme (aliment) (Almasad et al., 2011) en vue de satisfaire tous les besoins nutritionnels de la volaille en accord avec les objectifs de production (Pratiksha, 2011). Des étapes incluant la détermination de la quantité de nutriments que chaque ingrédient rendra disponible pour le métabolisme animal, le niveau de nutriments nécessaire pour que l'animal atteigne le niveau de production souhaité et la méthode de formulation de ces aliments, doivent être utilisés pour atteindre les objectifs (Pomar et al., 2009).

Des fermiers emploient des méthodes manuelles inefficaces, d'autres improvisent des démarches pour résoudre les problèmes de formulation d'aliment de volaille (Oladokun et Johnson, 2012). Dans les années 1950, avec le développement des programmations mathématiques via les ordinateurs, les spécialistes ont repris et développés des méthodes permettant de formuler un aliment à moindre coût (Pena et al., 2009). Ces méthodes ont permis de minimiser les coûts de production d'aliment ou du ratio coût aliment - gain de poids. Ce travail présente une revue des étapes et les méthodes manuelles et mathématiques de la formulation d'aliment de volaille.

ETAPES DE LA FORMULATION D'ALIMENT DE VOLAILLE

Connaissance de l'animal et détermination des besoins nutritionnels

Les besoins nutritionnels sont influencés par la génétique, le sexe, le poids vif, le stade physiologique, l'appétit et les facteurs environnementaux (température, densité...) (NRC, 1981). De ce fait il importe de bien identifier la catégorie de volaille à nourrir.

Les besoins nutritionnels peuvent être définis comme étant la quantité de nutriments nécessaires pour optimiser un facteur de production, tel que la vitesse de croissance ou la conversion alimentaire (Pomar et al., 2009). La vitesse de croissance, les performances de production de chair ou d'œuf, la formation du muscle ou de l'œuf (Moughan et al., 2000), le dépôt de graisse ou des éléments nutritifs dans les organes, la consommation alimentaire sont autant des caractéristiques à définir avant de déterminer le besoin (Sakomura et al., 2005). Pour la réussite de la formulation, la détermination des besoins en plusieurs éléments nutritifs essentiels est donc primordiale. Au nombre de ces éléments, il y a l'énergie métabolisable, les protéines brutes, les acides aminés, le calcium et le phosphore (...). Traditionnellement, les besoins nutritionnels des volailles sont publiés sous forme de tables (INRA, 1989; NRC, 1994).

\section{Détermination des valeurs nutritives des ingrédients disponibles}

Pour le besoin de formulation d'aliment, la réalisation d'une base de données sur la composition chimique, les caractéristique physiques et la digestibilité des ingrédients utilisables en alimentation des animaux est nécessaire (Kaushik, 2000). Il existe des bases de données (tables) des compositions chimiques des ingrédients pour aliment volailles (NRC, 1994 ; FAO et al, 2015) qui sont mises à jour continuellement (Kaushik, 2000). 
Cependant, les matières premières utilisées pour la formulation des aliments dépendent du pays ou de la région en fonction des potentialités des ingrédients (Moughan et al., 2000 ).

Dans ce contexte, il est recommandé au formulateur de constituer sa propre base de données en fonction des ressources alimentaires disponibles (Kaushik, 2000).

Le nombre d'éléments nutritifs à considérer pour la formulation varie, mais les plus utilisés pour la formulation et le suivi des performances sont l'énergie métabolisable, les protéines brutes (Moughan et al., 2000), les acides aminés (Sterling et al., 2005), le calcium et le phosphore (Kaushik, 2000). Ces éléments doivent être de même nature que ceux considérés dans la définition des besoins nutritionnels des volailles à nourrir.

\section{Seuil d'incorporation des ingrédients et facteurs antinutritionnels}

En plus de la composition chimique et des valeurs nutritives des ingrédients, des connaissances sur le seuil d'incorporation de chaque ingrédient et les facteurs de variation de la valeur nutritive sont déterminants pour la réussite de la formulation d'aliment.

Les limites maximale et minimale de chaque ingrédient doivent être connues afin d'éviter la toxicité, le déséquilibre alimentaire, les interférences avec d'autres éléments nutritifs, la sous consommation liée à l'inappétence ou même la pollution de l'environnement après excrétion par l'urine et les fientes (Moughan et al., 2000).

Plusieurs ingrédients sont mélangés pour formuler l'aliment volaille, il est important de prendre en compte les facteurs de variation liés au mélange ; spécifiquement, la somme des facteurs antinutritionnels qui peuvent s'y trouver. En effet, leurs présences réduisent la digestibilité et l'absorption des nutriments (Moughan et al., 2000 ), inhibent les enzymes, provoquent les lésions de foie et de l'intestin (Pimpukdee et al., 2004) ou entraînent un retard de croissance chez les poulets (Miazzo et al., 2000).

\section{Formulation proprement dite}

Cette étape consiste à réaliser des calculs afin de déterminer la combinaison de différents ingrédients qui couvre au mieux les besoin recommandés pour la catégorie animale. Les aspects d'efficience sont intégrés dans certaines méthodes pour l'obtention d'un aliment à moindre coût.

\section{METHODES MANUELLES DE FORMULATION D'ALIMENT \\ Méthode carré de Pearson}

C'est une méthode qui détermine la proportion de deux ingrédients à mélanger pour satisfaire le besoin d'un nutriment (Rosshairy et al., 2010). Elle s'effectue avec le carré de Pearson ci-après (Lee, 2009).

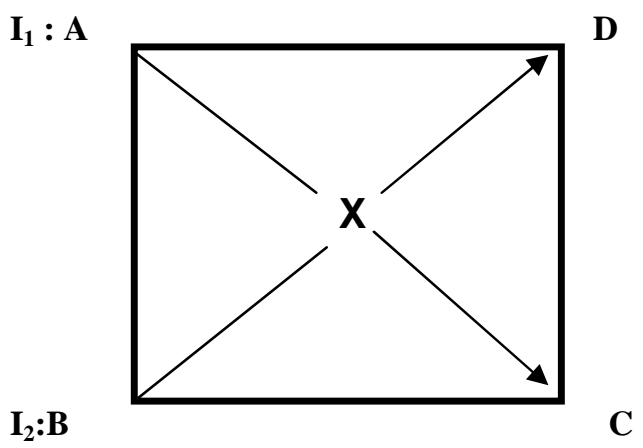

En $\mathbf{X}$ la solution désirée: le besoin nutritionnel à satisfaire

En $\mathbf{A}$ et $\mathbf{B}$ les teneurs en nutriment des deux sources d'ingrédients $\mathbf{I}_{\mathbf{1}}$ et $\mathbf{I}_{\mathbf{2}}$ pour satisfaire le besoin nutritionnel $\mathbf{X}$;

- $\mathbf{C}$ représente la différence entre $\mathbf{A}$ et $\mathbf{X}$ sans tenir compte de signe; c'est la part de l'ingrédient $\mathbf{I}_{\mathbf{2}}$ dans le mélange ;

- D représente la différence entre $\mathbf{B}$ et $\mathbf{X}$ sans tenir compte de signe; c'est la part de l'ingrédient $\mathbf{I}_{\mathbf{1}}$ dans le mélange.

La proportion (\%) d'ingrédient $\mathbf{I}_{1}$ contenant le nutriment A s'obtient par $\frac{|C|}{|C|+|D|} * 100$

La proportion (\%) d'ingrédient $\mathbf{I}_{\mathbf{2}}$ contenant le nutriment B s'obtient par $\frac{|D|}{|C|+|D|} * 100$

Cette méthode est relativement simple et facile à suivre (Olusayo et al., 2013). Roush 
et al. (1996) l'ont utilisée pour trouver un équilibre de $23 \%$ de protéine dans l'aliment à partir du maïs et du tourteau de soja. La méthode Carrée de Pearson peut s'effectuer avec le tableur Excel (Onwurah, 2012).

Les limites de cette méthode résident de fait qu'elle utilise seulement deux ingrédients pour déterminer un seul besoin (Afolayan et Afolayan, 2008). Toutefois, il est possible d'utiliser des prémélanges d'ingrédients afin d'accroître le nombre d'ingrédient dans l'aliment formulé. Elle ne peut pas être utilisée dans des formulations avec plusieurs besoins à satisfaire (Rosshairy et al., 2010).

\section{Méthode d'équations algébriques simultanées}

C'est une méthode alternative à celle de carré de Pearson en utilisant une équation algébrique simple (Afolayan et Afolayan, 2008). Elle permet de satisfaire un besoin nutritionnel en faisant la combinaison de deux ingrédients (Olusayo et al., 2013) à travers un système d'équation(Lee, 2009).

$$
\left\{\begin{array}{c}
a X+h Y=\text { hesnin à satisfrire } \\
X+Y=100 \text { de besoin à satisfaire }
\end{array}\right.
$$

- $\mathbf{X}$ et $\mathbf{Y}$ sont les inconnues de l'équation et représentent les pourcentages des deux sources d'ingrédients à mélanger ;

- $\quad \mathbf{a}$ et $\mathbf{b}$ sont les teneurs en nutriment contenues dans les deux ingrédients respectifs. C'est une méthode simple. Elle peut être utilisée par les fermiers (Olusayo et al., 2013). Elle peut comporter plusieurs ingrédients pour satisfaire un besoin (Afolayan et Afolayan, 2008). Ses limites sont qu'elle ne peut satisfaire qu'un seul besoin et ne peut pas être utilisée dans une formulation avec plusieurs besoins à satisfaire (Rosshairy et al., 2010).

\section{Méthode matricielle}

Cette méthode permet de trouver la solution conduisant à couvrir deux besoins nutritionnels avec deux ingrédients (Olusayo et al., 2013). Elle peut être même utilisée pour plusieurs besoins avec plus de deux ingrédients (Afolayan et Afolayan, 2008). Roush (1982) l'a utilisé pour satisfaire le besoin du poulet de chair en énergie, protéine, calcium et phosphore en mélangeant le maïs, le tourteau de soja, le phosphate mono calcique et le calcaire. Le système d'équation qu'il a utilisé est le suivant :

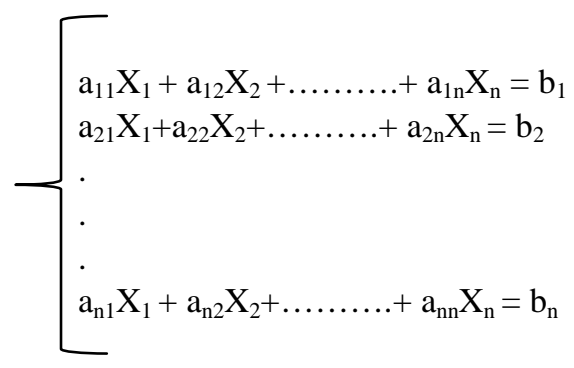

Avec a les teneurs en élément nutritif, $\mathrm{X}$ les proportions à trouver et $\mathrm{b}$ les besoins à satisfaire.

C'est une méthode efficiente permettant de définir le niveau énergétique désiré et d'autres nutriments avec les équations algébriques (Rosshairy et al., 2010). Elle a cependant l'inconvénient d'être complexe et demande beaucoup de temps pour sa résolution si le besoin nutritionnel dépasse deux éléments.

\section{Méthode des essais et erreurs}

Cette méthode est la plus populaire et utilisée pour la formulation des aliments volailles (Afolayan et Afolayan, 2008). Elle permet de satisfaire le besoin de la volaille en manipulant les valeurs nutritives des ingrédients en fonction des pourcentages fixés (Olusayo et al., 2013). Elle peut se faire manuellement ou en utilisant les tableurs de calcul comme Excel, Lotus123 ou Quattro pro (Rosshairy et al., 2010).

Cette méthode permet de satisfaire tous les besoins des poulets mais, a l'inconvénient d'être fastidieuse et prend du temps avant d'arriver à une solution satisfaisante (Afolayan et Afolayan, 2008). 


\section{METHODES DE PROGRAMMATION MATHEMATIQUE \\ Programmation linéaire}

La programmation linéaire est une méthode statistique pour choisir, repartir et évaluer des ressources limitées et plusieurs contraintes afin d'obtenir une fonction algébrique linéaire. Elle détermine la façon d'avoir un résultat sous la forme d'un système d'équation mathématique (Olorunfemi, 2007). La programmation linéaire fournie une méthode de formulation efficiente des aliments. Elle permet de minimiser le coût de l'aliment en trouvant un équilibre entre le pourcentage, la valeur nutritive et les contraintes de l'ingrédient utilisé pour la formulation (Al-Deseit, 2009).

Les principes de la programmation linéaire à moindre coût en tenant compte des contraintes sont donnés par les équations suivantes :

$\mathrm{Z}=\sum_{\mathrm{i}=1}^{\mathrm{n}}$ Cixi pour les coûts minimums
$\sum_{\mathrm{i}=1}^{\mathrm{n}}$ aiXi $\geq \mathrm{Bi}$ avec $\mathrm{Xi} \geq 0$ pour les contraintes

Avec Z : coût total de l'aliment ; $\mathrm{Ci}$ : le coût unitaire de l'ingrédient rapporté au $\mathrm{Kg}$; Xi : la quantité de l'ingrédient $\mathrm{i}$ dans l'aliment en $\mathrm{Kg}$; ai : les valeurs nutritives de l'ingrédienti et $\mathrm{Bi}$ les niveaux de besoin à satisfaire pour chaque ingrédient (Olorunfemi, 2007; AlDeseit, 2009 ; Almasad et al., 2011 ; Baghat et Bajaj, 2014). Thomson et Nolan (2001) ont utilisé la fonction Solveur d'Excel pour concevoir un tableur d'enseignement et de formulation d'aliment des volailles à moindre coût par programmation linéaire.

Certaines conditions doivent être vérifiées avant l'utilisation des résultats de la programmation linéaire (Olorunfemi, 2007 ; Al-Deseit, 2009) qui sont résumés aux principes de :

Certitude: les valeurs numériques et les contraintes des ingrédients doivent être connues dans l'équation et ne doivent pas changer au cours de la formulation ;
Divisibilité : la proportion de chaque ingrédient doit être un nombre numérique et représenter une fraction de la valeur totale ;

Additivité : la somme des proportions des ingrédients utilisés doivent correspondre à la somme des quantités de différents ingrédients dans l'aliment ;

Linéarité : il doit y avoir une relation entre la proportion est la quantité de chaque ressources utilisées ;

Non négativité : toutes les proportions doivent être supérieures à zéro.

\section{Programmation non linéaire}

Les tables de composition chimique des ingrédients ne présentent que les valeurs moyennes des paramètres nutritionnels. Elles ne représentent pas la totalité des échantillons (Pena et al., 2009). Si la programmation linéaire permet d'optimiser une formule alimentaire, elle ne prend pas en compte les variabilités des ingrédients (Guevara, 2004). Ces contraintes sont reconnues par les chercheurs comme un problème pour la formulation des aliments et la programmation non linéaire apparaît comme une solution pouvant résoudre ces variabilités.

Elle est plus utilisée pour décrire les interdépendances qui existent entre le niveau d'incorporation d'un ingrédient ou les relations entre les valeurs nutritives (énergie, protéine, calcium...) d'un aliment et les performances induites ou la marge bénéficiaire dégagée sous la forme d'une fonction mathématique (Heydari, 2014). Afrouziyeh et al. $(2010,2011)$ ont utilisé le solveur d'Excel pour optimiser la production des œufs par programmation non linéaire et pour déterminer les relations qui existent entre le poids d'œuf et la densité énergétique de l'aliment pondeuse durant la phase 1 (24 à 32 semaines d'âge) et la phase 2 (32 à 44 semaines d'âge).

\section{Programmation multi objective}

La programmation multi objective a été utilisée en 1983 pour résoudre le déséquilibre nutritionnel en alimentation humaine 
(Rosshairy et al., 2010). En alimentation animale, la programmation multi objective est utilisée pour prendre en compte la formulation à moindre coût et le déséquilibre nutritionnel des aliments (Pena et al., 2009).

Le model utilisé en programmation multi objective est comparable à celui de programmation linéaire en tenant en compte le déséquilibre alimentaire (Rosshairy et al., 2010). La variation de besoin nutritionnel est modélisée selon Zhang et Roush, (2002) par :

$\sum_{i=1}^{n} V i j X i^{2}$

Où : $\mathrm{V}$ est la variance du nutriment $\mathrm{j}$ dans l'ingrédient $\mathrm{i}$; $\mathrm{Xi}$ le pourcentage de l'ingrédient i dans l'aliment. Quand beaucoup d'ingrédients sont utilisés, certains pourcentages deviennent petits, d'où l'utilisation de la variance des nutriments et du carré des ingrédients (Zhang et Roush, 2002).

Zhang et Roush (2002) ont utilisé la technique de programmation multi objective pour prendre en compte les contraintes liées à la variation de besoin nutritionnel entre poulets de chair en trouvant un optimum dans le groupe.

\section{Programmation quadratique}

C'est une programmation qui permet de maximiser le profit en utilisant les besoins nutritionnels. Cette méthode permet de choisir la formule alimentaire qui minimise le coût de l'aliment dans une série de formules qui satisfait à toutes les contraintes. Elle utilise l'énergie et les protéines comme facteurs qui permettent de faire le choix (Rosshairy et al., 2010).

Miller et al. (1986) ont proposé le model de programmation quadrique pour optimiser l'aliment des poulets de chair. Les fonctions d'optimisation sont les concentrations énergétiques et protéiques de l'aliment selon le model :

$$
\sum_{j} r j X j \quad j=1 \ldots \ldots n_{r} \quad \text { pour les coûts }
$$

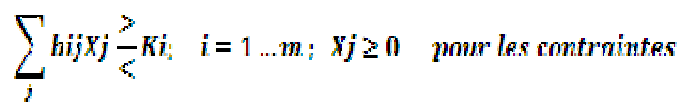

Où : rj le coût de l'aliment $\mathrm{j} ; \mathrm{Xj}$ la quantité de l'ingrédient $\mathrm{j}$; bijla part du nutriment $\mathbf{i}$ dans l'ingrédient $\mathrm{j}$ et ki le besoin en nutriment i. K représente l'énergie et les protéines.

\section{Conclusion}

La formulation d'aliment pour volaille est une démarche délicate et précise. Elle nécessite la détermination des besoins nutritionnels de l'animal à nourrir, la connaissance des compositions chimique et nutritionnels des ingrédients, la définition du seuil d'incorporation et l'utilisation d'une méthode de calcul pour aboutir aux objectifs. Les méthodes manuelles permettent de trouver une solution avec peu d'ingrédients. Les méthodes mathématiques permettent quant à elles de faire une formulation à moindre coût, de chercher les relations entre performance et valeur nutritive des ingrédients et de réduire le déséquilibre alimentaire. Bien que les méthodes manuelles soient simples et populaires, elles ne permettent pas de prendre en compte toutes les contraintes nutritionnelles et économiques. Par contre, les méthodes mathématiques qui prennent en charge ces contraintes nécessitent une certaine technicité et ne sont donc pas accessibles à tous. La méthode la plus appropriée permettant de répondre aux préoccupations nutritionnelles, économiques et d'accessibilité serait la programmation linéaire.

\section{REMERCIEMENTS}

Les auteurs adressent leurs sincères remerciements et leur profonde gratitude à tous ceux qui ont contribué à la réalisation de ce présent article, en particulier au Programme de Productivité Agricole en Afrique de l'Ouest du Niger (PPAAO-Niger), à l'Université Abomey Calavi (UAC) du Benin et à l'Institut National de la Recherche Agronomique du Niger (INRAN).

\section{REFERENCES}

Afolayan MO, Afolayan M. 2008.Nigeria Oriented Poultry Feed Formulation 
Software Requirements. Journal of Applied Sciences Research, 4(11): 15961602.

Afrouziyeh M, Shivazad M, Chamani M, Dashti G, Amirdahri S. 2011.Use of nonlinear programming to determine the economically optimal energy density in laying hens diet during phase 2. Journal of Applied Poultry Research, 20: 50-55. DOI: 10.3382/japr.2010-00205

Afrouziyeh M, Shivazad M, Chamani M, Dashti G. 2010. Use of nonlinear programming to determine the economically optimal energy density in laying hens diet during phase 1. African Journal of Agricultural Research, 5(20): 2770-2777.

Al-Deseit B. 2009. Least-Cost Broiler Ration Formulation Using Linear Programming Technique.Journal of Animal and Veterinary Advances, 8(7): 1274-1278. DOI: javaa.2009.1274.1278

Almasad M, Altahat E, AL-Sharafat A. 2011. Applying Linear Programming Technique to Formulate Least Cost Balanced Ration for White Eggs Layers in Jordan. International Journal of Empirical Research, 1(1): 112-120. DOI: 10. 5897/AJAR

Bhagat AA, Bajaj VH. 2014. Animalfeed formulation by using linear programming technique. International Journal of Statistika and Mathematika, 12(2): 101104. www.statperson.com, DOI: 14 December 2014

FAO, INRA, CIRAD, AFZ. 2015. Feedipedia: on-line encyclopedia of animal feeds. Disponible en ligne le 03/06/2015:http://www.feedipedia.org/

Guevara VR. 2004. Use of nonlinear programming to optimize performance response to energy density in broiler feed formulation. Poultry Science, 83: 147 151. DOI : $10.1093 / \mathrm{ps} / 83.2 .147$

INRA (Institut National de la Recherche Agronomique). 1989. Alimentation des animaux monogastriques: Porc, Lapin, Volailles, $2^{\text {nd }}$ édn. INRA: France
Kaushik SJ. 2000. Feed formulation, diet development and feed technology. In Recent advances in Mediterranean aquaculture finfish Species Diversification. Zaragoza: CIHEAM; 4351.

Heydari SG. 2014. Effect of linear and random non-linear Programming feed formulating on performance of broilers. Journal of Novel Applied Sciences, 3(12): 1426-1429.

Lee IC. 2009. Animal Nutrition Handbook (Second Revision). 552p Disponible en ligne le 25/5/2015: https://umkcarnivores3.files.wordpr ess.com/2012/02/animal-nutrition2.pdf

Miazzo R, Rosa CAR, De Queiroz Carvalho EC, Magnoli C, Chiacchiera SM, Palacio G, Saenz M, Kikot A, Basaldella E, Dalcero A. 2000. Efficacy of synthetic zeolite to reduce the toxicity of aflatoxin in broiler chicks. Poultry Science, 79: 1-6. DOI:10.1093/ps/79.1.1

Miller BR, Arraes RA, Pesti GM. 1986. Formulation of Broiler Finishing Rations by Quadratic Programming.Southern Journal of Agricultural Economics, 18: 141-150. DOI: http://dx.doi.org/10.1017/ S0081305200005446

Moughan PJ, Verstegen MWA, VisserReyneveld MI. 2000. Feed Evaluation: Principles and Practice. Wageningen Press: Wageningen, Netherlands.

NRC (National Research Council). 1994. Nutrient Requirements of Poultry $\left(9^{\text {th }}\right.$ edn). National Academy Press: Washington, D.C.

NRC (National Research Council). 1981. Effect of Environment on Nutrient Requirements of Domestic Animals. National Academy Press: Washington, D.C.

Oladokun VO, Johnson A. 2012. Feedformulation problem in Nigerian poultry farms: a mathematical programming approach. American Journal of Scientific and Industrial 
Research, 3(1): 14-20. $\quad$ DOI: 10.5251/ajsir.2012.3.1.14.20

Olorunfemi TOS. 2007. Linear programming application to utilization of duckweed (Lemna Paucicostata) in least-cost ration formulation for broiler finisher. Journal of Applied Sciences, 6(9): 1909-1914. DOI: $10.3923 /$ jas.2006.1909.1914

Olusayo OE, Olusesan AB, Adesola AG. 2013. Review of Livestock Feed Formulation Techniques. Journal of Biology, Agriculture and Healthcare, 3(4): 60-77.

Onwurah FB. 2012. Excel feed formulation and feeding models; Indian J. Edu. Inf. Manage, 1(2): 88-92.

Pena T, Lara P, Castrodeza C. 2009. Multiobjective Stochastic Programming for Feed Formulation. Journal of the Operational Research Society, 60: 1738 1748. DOI: $10.1057 /$ jors.2008.106

Pimpukdee K, Kubena LF, Bailey CA, Huebner HJ, Afriyie-Gyawu E, Phillips TD. 2004. Aflatoxin-Induced Toxicity and Depletion of Hepatic Vitamin A in Young Broiler Chicks: Protection of Chicks in the Presence of Low Levels of NovaSil PLUS in the Diet.Poultry Science, 83: 737-744. DOI : 10.1093/ps/83.5.737

Pomar C, Dubeau F, Van Milgen J. 2009. La détermination des besoins nutritionnels, la formulation multicritère et l'ajustement progressif des apports de nutriments aux besoins des porcs: des outils pour maitriser les rejets d'azote et de phosphore. INRA Productions Animales, 22(1): 49-54.

Pratiksha S. 2011. Comparaison of Linear and Nonlinear Programing Techniques for Animal Diet. Applied Mathematics, 1(2):
106-108. DOI: $\quad$ 10.5923/j.am. 20110102.17

Rosshairy AR, Chooi-Leng A, Razamin R. 2010. Investigating Feed Mix Problem Approaches: An Overview and Potential Solution. World Academy of Science, Engineering and Technology, 70: 467475.

Roush WB. 1982. Using the Matrix Algebra Capabilities of the TI59 Calculator for Teaching Ration Formulation. Poultry Science, 61: 2153-2156. DOI: 10.3382/ps.0612153

Roush WB, Terri LC, Fushan Z. 1996.Computer formulation observations and caveats. Journal of Applied Poultry Research, 5: 116-125. DOI: 10.1093/japr/5.2.116

Sakomura NK, Longo FA, Oviedo-Rondon EO, Boa-Viagem C, Ferraudo A. 2005. Modeling Energy Utilization and Growth Parameter Description for Broiler Chickens. Poultry Science, 84: 13631369. DOI: $10.1093 / \mathrm{ps} / 84.9 .1363$

Sterling KG, Vedenov DV, Pesti GM,Bakalli RI. 2005. Economically Optimal Dietary Crude Protein and Lysine Levels for Starting Broiler Chicks. Poultry Science, 84: 29-36. DOI: $10.1093 / \mathrm{ps} / 84.1 .29$

Thomson E, Nolan J. 2001. UNEForm: a powerful feed formulation spreadsheet suitable for teaching or on-farm formulation. Animal feed Science and Technology, 91: 233-240. DOI: 10.1016/S0377-8401(01)00210-3

Zhang F, Roush WB. 2002. MultipleObjective (Goal) Programming Model for Feed Formulation: An Example for Reducing Nutrient Variation. Poultry Science, 81: 182-192. DOI:10.1093/ps/81.2.182 